\title{
Grey wolf optimizer for identification of liquid slosh behavior using continuous-time Hammerstein model
}

\author{
Mohd Ashraf Ahmad ${ }^{1}$, Zulkifli Musa ${ }^{2}$, Mohd Helmi Suid ${ }^{3}$, Mohd Zaidi Mohd Tumari ${ }^{4}$ \\ ${ }^{1,2,3}$ Faculty of Electrical and Electronics Engineering Technology, University Malaysia Pahang, Pahang, Malaysia \\ ${ }^{4}$ Faculty of Electrical \& Electronic Engineering Technology, Universiti Teknikal Malaysia Melaka, Melaka, Malaysia
}

\section{Article Info \\ Article history: \\ Received Oct 14, 2019 \\ Revised Dec 28, 2019 \\ Accepted Jan 24, 2020 \\ Keywords: \\ Continuous-time \\ Hammerstein model \\ Metaheuristic algorithm \\ Slosh nature \\ System identification}

\begin{abstract}
This paper presents the identification of liquid slosh plant using the Hammerstein model based on Grey Wolf Optimizer (GWO) method. A remote car that carrying a container of liquid is considered as the liquid slosh experimental rig. In contrast to other research works, this paper consider a piece-wise affine function in the nonlinear function of the Hammerstein model, which is more generalized function. Moreover, a continuous-time transfer function is utilized in the Hammerstein model, which is more suitable to represent a real system. The GWO method is used to tune both coefficients in the nonlinear function and transfer function of the Hammerstein model such that the error between the identified output and the real experimental output is minimized. The effectiveness of the proposed framework is assessed in terms of the convergence curve response, output response, and the stability of the identified model through the bode plot and pole zero map. The results show that the GWO based method is able to produce a Hammerstein model that yields identified output response close to the real experimental slosh output.
\end{abstract}

This is an open access article under the CC BY-SA license.



\section{Corresponding Author:}

Mohd Ashraf Ahmad,

Faculty of Electrical and Electronics Engineering Technology,

University Malaysia Pahang, 26600, Pekan, Pahang, Malaysia.

Email:mashraf@ump.edu.my

\section{INTRODUCTION}

Nowadays, liquid slosh inside a cargo always happens in many situations. For example, ships with liquid container carriers are at high risk of generating sloshing load during operation [1]. In the metal industries, high oscillation can spill molten metal that is dangerous to the operator [2]. Meanwhile, sloshing of fuel and other liquids in moving vehicles may cause instability and undesired dynamics [3]. Hence, it is necessary to completely study the behavior of this residual slosh induced by the container motion. One may study the behavior of liquid slosh through developing the exact mathematical model of liquid slosh. So far, many researchers focus on the first principle approach to model the slosh behavior, while there are few literatures to discuss it from the perspective of nonlinear system identification approach.

On the other hand, block oriented nonlinear system identification has became a popular techniques to model a complex plants. The block oriented nonlinear model can be classified into three categories, which are Hammerstein model, Wiener model and Hammerstein Wiener model. In particular, Hammerstein model is a model that consists of a nonlinear function followed by linear dynamic sub-plant, while Wiener model consists of a linear dynamic sub-plant followed by nonlinear function, and finally, Hammerstein-Wiener model contains a linear dynamic sub-plant inserted between two or more nonlinear functions in series. Among these three block oriented models, Hammerstein model is famous due to its simple model structure 
and it has been widely used for nonlinear system identification. Specifically, the Hammerstein model has been applied to model a real plant such as Solid Oxide fuel cell [4], bidirectional DC motor [5], oxygen uptake estimation [6], stretch reflex dynamics [7], turntable servo system [8], pneumatic muscle actuators [9], amplified piezoelectric actuators [10] and multi-axis piezoelectric micro positioning stages [11]. On the other hand, there are many tools that have been utilized to identify the Hammerstein model. There are the iterative method [12-14], the subspace method [15-17], the least square method [18], the blind approach [19] and the parametric instrumental variables method [20]. Moreover, many also consider the optimization tools for Hammerstein model, such as Bacterial Foraging algorithm [21], Cuckoo search algorithm [22], Particle Swarm optimization [23], and Genetic algorithm [24]. Based on the above literature, several limitations are ineluctable in their works, which are:

a. Most of the Hammerstein models used in their study are based on discrete-time model, while many real plants can be easily represented in continuous-time model.

b. Almost all the methods assume a known structure of nonlinear function, which consists of several basis functions.

Though, our proposed work can solve a more general class of continuous-time Hammerstein model by assuming an unknown structure of nonlinear function. In particular, a piece-wise affine function is adopted with so many basis functions. Due to the introduction of the piece-wise affine function, a high dimensional design parameter tuning is considered in this study, which make the identification problem more complex. On the other hand, Grey Wolf Optimizer (GWO) [25] has become a top notch optimization algorithm which has solved various types of engineering problems [25-27]. To the best of our knowledge, there are still few works to discuss on the GWO for identification of Hammerstein model. Thence, it motivates us to see the effectiveness of the GWO in modeling the liquid slosh plant from the real experimental data.

This paper presents the identification of liquid slosh plant using the Hammerstein model based on GWO method. A remote car carrying a container of liquid is considered as the liquid slosh experimental rig. The GWO method is used to tune both coefficients in the nonlinear function and transfer function of the Hammerstein model such that the error between the identified output and the real experimental output is minimized. The effectiveness of the proposed framework is assessed in terms of the convergence curve response, output response, and the stability of the identified model through the bode plot and pole zero map.

\section{RESEARCH METHOD}

This section explains on how the identification of liquid slosh based on Hammerstein model and GWO based method is performed. Firstly, the experimental setup of liquid slosh plant is explained. Here, it is shown on how the input and output data are generated. Secondly, we show the procedure to identify the model of liquid slosh plant using the Hammerstein based Grey Wolf Optimizer.

\subsection{Liquid slosh experimental rig}

In this study, a mobile liquid slosh plant is considered to replicate real situation of a moving container carrying liquid, as shown in Figure 1. In particular, a remote control car is used to carry a small tank filled with liquid. The tank is also equipped with four plastic wheels so that it can move smoothly as shown in Figure 1(a). Moreover, three accelerometer sensors (ADXL335) that are floated on the surface of liquid are used to measure liquid oscillation as shown in Figure 1(b). For simplicity of our study, the liquid slosh data from only one of the sensor is recorded and only z-axis output data is considered. Figure 2 shows a general schematic diagram of liquid slosh experimental rig.

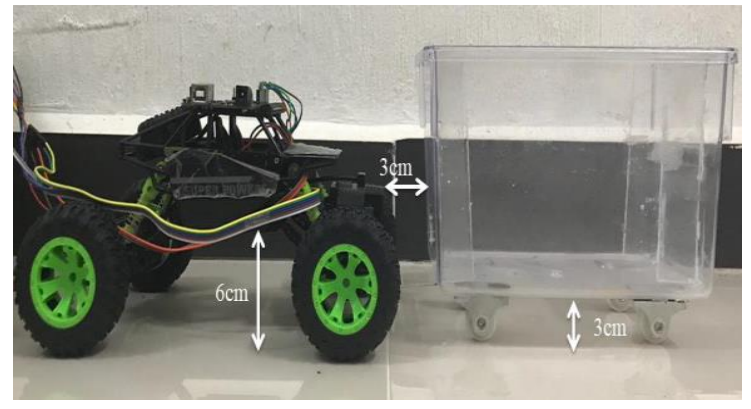

(a)



(b)

Figure 1. Liquid slosh experimental rig, (a) side view, (b) plan view 


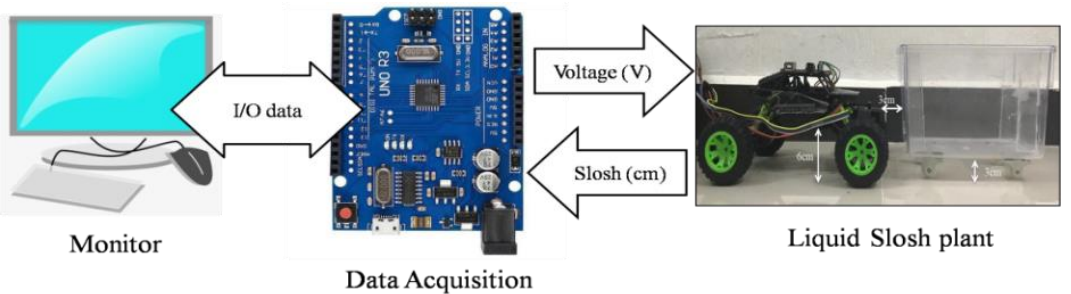

Figure 2. Schematic diagram of liquid slosh experimental rig

In particular, an Arduino UNO is used as a data acquisition platform to process the input and output data. Here, we generate a voltage from the Arduino UNO to the remote car and concurrently the Arduino UNO also will acquire the slosh data from the accelerometer. Both the input and output data can be monitored and analyzed from the personal computer using the LabView software. In order to identify the model of liquid slosh, the remote car is required to move to a certain distance and suddenly stop to generate a liquid oscillation or slosh inside the tank. Thence, we apply the input voltage as shown in Figure 3 to move the remote car. Concurrently, the liquid slosh data is recorded as shown in Figure 4 . These two data are then used to develop the Hammerstein model based GWO, which is discussed in the next section.

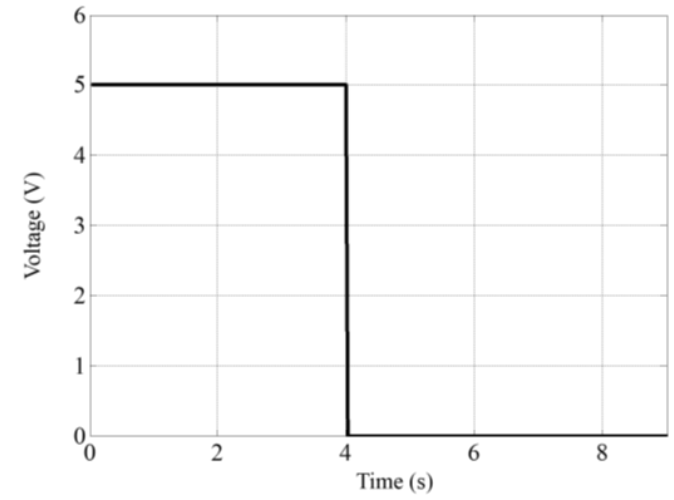

Figure 3. Input voltage applied to the remote car

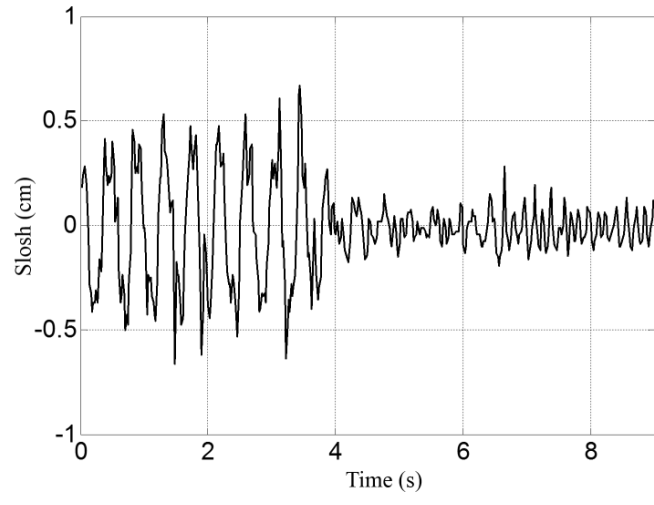

Figure 4. Output slosh from the accelerometer

\subsection{Identification of liquid slosh using Hammerstein based grey wolf optimizer}

In this section, the proposed Grey Wolf Optimizer (GWO) for identification of liquid slosh plant in Section 2.1 based on Hammerstein model is presented. Firstly, a problem formulation to identify the liquid slosh plant is explained. Then, it is shown on how to apply the GWO method to identify the liquid slosh based on Hammerstein model. Figure 5 shows a complete block diagram to identify the liquid slosh model in Section 2.1. The proposed Hammerstein model consists of nonlinear function $h(u)$ followed by the transfer function $G(s)$. The nonlinear function is a piece-wise affine function given by

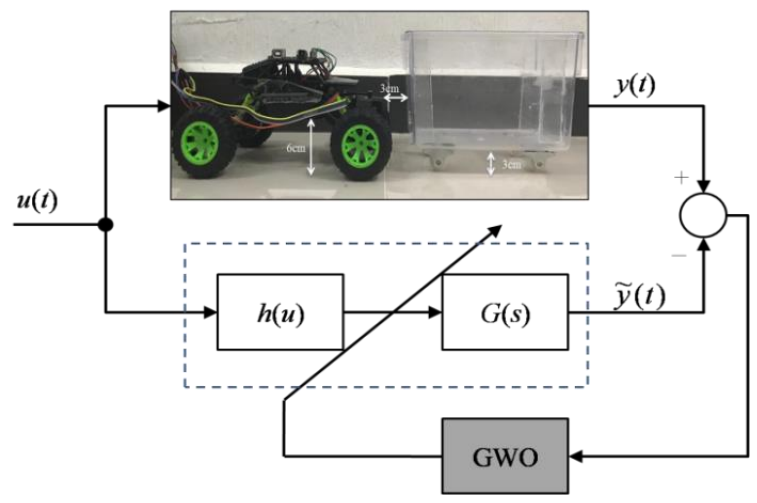

Figure 5. Block diagram of Hammerstein model based GWO 


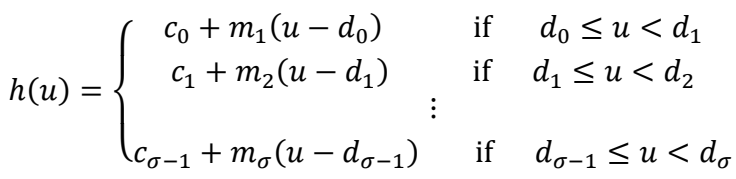

and the transfer function $G(s)$ is given by

$$
G(s)=\frac{B(s)}{A(s)}=\frac{s^{m}+b_{m-1} s^{m-1}+\cdots+b_{0}}{a_{m} s^{m}+a_{m-1} s^{m-1}+\cdots+a_{0}}
$$

In (1), the symbol $m_{i}=\left(c_{i}-c_{i-1}\right) /\left(d_{i}-d_{i-1}\right)(i=1,2, \ldots \sigma)$ are the segment slope with connecting input and output points as $d_{i}(i=0,1, \ldots \sigma)$ and $c_{i}(i=0,1, \ldots \sigma)$, respectively. For simplicity of notation, let $\boldsymbol{d}=\left[d_{0}, d_{1}, \ldots, d_{\sigma}\right]^{\mathrm{T}}$ and $\boldsymbol{c}=\left[c_{0}, c_{1}, \ldots, c_{\sigma}\right]^{\mathrm{T}}$. The input of the real liquid slosh plant and the identified model is defined by $u(t)$, while the output of the real liquid slosh plant and the identified model are denoted by $y(t)$ and $\tilde{y}(t)$, respectively. Thence, the expression of the identified output can be written as

$$
\tilde{y}(t)=G(s) h(u(t))
$$

Moreover, several assumptions are adopted in this work, which are:

a. The order of the polynomial $A(s)$ and $B(s)$ are assumed to be known

b. The nonlinear function $h(u(t))$ is one-to-one map to the input $u(t)$ and the values of $d_{i}(i=1,2, \ldots \sigma)$ are pre-determined according to the response of input $u(t)$.

Next, let $t_{s}$ be a sampling time for the real experimental input and output data $(u(t), y(t))$ $\left(t=0, t_{s}, 2 t_{s}, \ldots, N t_{s}\right)$. Then, in order to accurately identify the liquid slosh model, the following objective function in (4) is adopted in this study:

$$
E(G, h)=\sum_{\eta=0}^{N}\left(y\left(\eta t_{s}\right)-\tilde{y}\left(\eta t_{s}\right)\right)^{2}
$$

Finally, our problem formulation can be described as follows

Problem 1. Based on the given real experimental data $(u(t), y(t))$ in Figure 1, find the nonlinear function $h(u)$ and the transfer function $G(s)$ such that the objective function in (4) is minimized.

Furthermore, it is shown on how to apply the GWO in solving Problem 1. For simplicity, let the design parameter of Problem 1 is defined as $\boldsymbol{x}=\left[\begin{array}{llllllllllll}b_{0} & b_{1} & \ldots & b_{m-1} & a_{0} & a_{1} & \ldots & a_{m} & c_{0} & \ldots & c_{\sigma}\end{array}\right]^{T}$, where the elements of the design parameter are the coefficients of both the nonlinear function and the transfer function of the continuous-time Hammerstein model. In GWO framework, let $x_{i}(i=1,2, \ldots, M)$ be the design parameter of each agent $i$ for $M$ total number of agents. Then, consider $x_{i j}(j=1,2, \ldots, D)$ be the $j$-th element of the vector $x_{i}(i=1,2, \ldots, M)$, where $D$ is the size of the design parameter. Thence, by adopting objective function in (4), a minimization problem is expressed as

$$
\arg \min _{x_{i}(1), x_{i}(2), \ldots} E\left(x_{i}(k)\right)
$$

for iterations $k=1,2, \ldots$, until maximum iteration $k_{\max }$. Finally, the procedure of the GWO in solving Problem 1 is shown below:

- Step 1: Determine the total number of agents $M$ and the maximum iteration $k_{\max }$. Set $k=0$ and initialize the design parameter $x_{i}(0)(i=1,2, \ldots, M)$ according to the upper bound $\boldsymbol{x}_{\text {up }}$ and lower bound $\boldsymbol{x}_{\text {low }}$ values of the design parameter.

- Step 2: Calculate the objective function in (4) for each search agent $i$.

- Step 3: Update the values of the best design parameter $\boldsymbol{x}^{\alpha}$, the second best design parameter $\boldsymbol{x}^{\beta}$ and the third best design parameter $\boldsymbol{x}^{\delta}$ based on the generated objective function in Step 2.

- Step 4: For each agent, update the design parameter using the following equation:

$$
x_{1}(k+1)=\frac{x^{1}+x^{2}+x^{3}}{3}
$$

where

$$
\begin{aligned}
& x^{1}=x^{\alpha}-P \cdot\left|Q \cdot x^{\alpha}-x_{i}(k)\right|, x^{2}=x^{\beta}-P \cdot\left|Q \cdot x^{\beta}-x_{i}(k)\right|, \\
& x^{3}=x^{\delta}-P \cdot\left|Q \cdot x^{\delta}-x_{i}(k)\right|
\end{aligned}
$$


for $i=1,2, \ldots, M$. In (6), the vectors $\boldsymbol{P}$ and $\boldsymbol{Q}$ are expressed as follows:

$$
\begin{aligned}
& P=2 a \cdot r_{1}-a \\
& Q=2 \cdot r_{2}
\end{aligned}
$$

where $r_{1}$ and $r_{2}$ are the random vectors, where each element is generated independently from 0 to 1 , and each element of $a$ is linearly decreased from 2 to 0 over the course of iteration using the following equation:

$$
a=2\left(1-\frac{k}{k_{\max }}\right)
$$

Note that the vectors of $\boldsymbol{P}$ and $\boldsymbol{Q}$ in (8) and (9), which consists of random vectors $\boldsymbol{r}_{1}$ and $\boldsymbol{r}_{2}$, are generated independently between $\boldsymbol{x}^{1}, \boldsymbol{x}^{2}$ and $\boldsymbol{x}^{3}$.

- Step 5: After the maximum iteration is achieved, record the best design parameter $\boldsymbol{x}^{\alpha}$ and obtained the continuous-time Hammerstein model in Figure 1. Otherwise, repeat Step 2.

\section{RESULTSAND ANALYSIS}

In this section, the effectiveness of the GWO based method for identifying the liquid slosh system using continuous-time Hammerstein model is demonstrated. In particular, the convergence curve response of the objective function in (4), the bode plot and pole-zero mapping of linear function and the plot of nonlinear function, will be presented and analyzed in this study.

Based on the experimental setup in Section 2.1, the input response $u(t)$ as shown in Figure 3 is applied to the liquid slosh plant, and the output response $y(t)$ is recorded as shown in Figure 4 . Here, the input and output data are sampled at $t_{s}=0.02$ for $N=450$. In this study, the structure of $G(s)$ is selected as follows:

$$
G(s)=\frac{B(s)}{A(s)}=\frac{s^{3}+b_{2} s^{2}+b_{1} s+b_{0}}{a_{4} s^{4}+a_{3} s^{3}+a_{2} s^{2}+a_{1} s+a_{0}}
$$

After performing several preliminary testing on the given data $(u(t), y(t))$. Meanwhile, the input points for piece-wise affine function of $h(u(t))$ are given by $\boldsymbol{d}=[0,0.2,0.4,0.6,0.8,1,2,3,4,5]^{\mathrm{T}}$. The design parameter $x \in R^{18}$ with its corresponding transfer function and nonlinear function is shown in Table 1 . Next, the GWO algorithm is applied to tune the design parameter with initial values of design parameter are randomly selected between the upper bound $\boldsymbol{x}_{\text {up }}$ and lower bound $\boldsymbol{x}_{\text {low }}$ as shown in Table 1. Here, we choose the number of agents $M=40$ with maximum iterations $k_{\max }=5000$.

Table 1. Design parameter of liquid slosh plant

\begin{tabular}{ccccc}
\hline $\boldsymbol{x}$ & Coefficients & $\boldsymbol{x}_{\text {low }}$ & $\boldsymbol{x}_{\text {up }}$ & $\boldsymbol{x}^{\alpha}$ \\
\hline$x_{1}$ & $b_{2}$ & -5 & 35 & -4.8017 \\
$x_{2}$ & $b_{1}$ & -5 & 35 & 0.2657 \\
$x_{3}$ & $b_{0}$ & -5 & 35 & -0.0243 \\
$x_{4}$ & $a_{4}$ & -5 & 35 & -0.1119 \\
$x_{5}$ & $a_{3}$ & -2200 & -1 & -4.3214 \\
$x_{5}$ & $a_{2}$ & -2200 & -1 & -31.6384 \\
$x_{7}$ & $a_{1}$ & -2200 & -1 & -901.2917 \\
$x_{8}$ & $a_{0}$ & -2200 & -1 & -1280.4483 \\
$x_{9}$ & $c_{0}$ & -5 & 5 & -1.9997 \\
$x_{10}$ & $c_{1}$ & -5 & 5 & 0.3249 \\
$x_{11}$ & $c_{2}$ & -5 & 5 & 1.5968 \\
$x_{12}$ & $c_{3}$ & -5 & 5 & -0.0757 \\
$x_{13}$ & $c_{4}$ & -5 & 5 & -0.0099 \\
$x_{14}$ & $c_{5}$ & -5 & 5 & -0.0048 \\
$x_{15}$ & $c_{6}$ & -5 & 5 & -0.0251 \\
$x_{16}$ & $c_{7}$ & -5 & 5 & 0.0584 \\
$x_{17}$ & $c_{8}$ & -5 & 5 & 0.0216 \\
$x_{18}$ & $c_{9}$ & -5 & 5 & -2.2603 \\
\hline
\end{tabular}


Figure 6 shows the response of the objective function convergence after 5000 iterations to produce the best design parameter as shown in the final column of Table 1. It shows that the GWO based method is able to minimize the objective function in (4) and produce a quite close output response $y(t)$ as compared to the real output $\tilde{y}(\mathrm{t})$, which can be clearly seen in Figure 7 . Note that the identified output response tends to yield high oscillation when input is injected to the system and it start to attenuate when the input is zero, which is quite similar to the response of real experimental output.

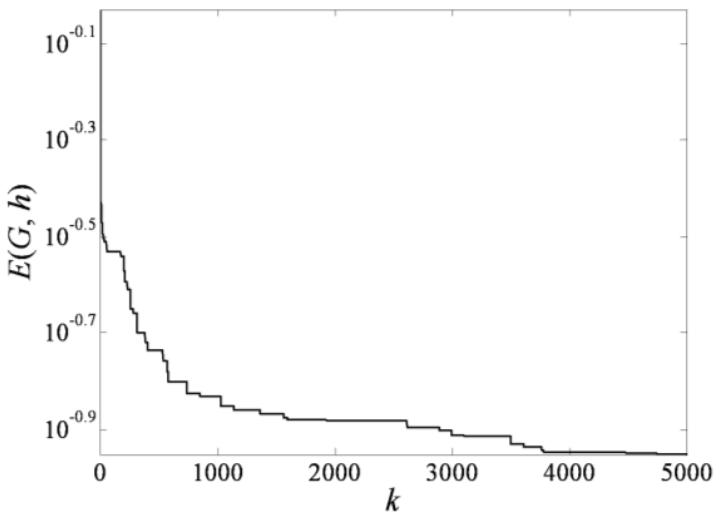

Figure 6. Convergence curve response



Figure 7. Response of the identified output $\tilde{y}(t)$ and real output $y(t)$

In the real experimental setup, we can say that the liquid slosh system is stable since the liquid slosh output is reduced gradually as $t \rightarrow \infty$. In order to validate our model regarding the stability, we use the bode diagram and the pole-zero map of the identified transfer function $G(s)$ as shown in Figures 8 and 9, respectively. From the bode diagram, it can be observed that a gain margin of $2.56 \mathrm{~dB}$ at $17.4 \mathrm{rad} / \mathrm{s}$ and phase margin of 4.18 degree at $16.5 \mathrm{rad} / \mathrm{s}$ are produced, which indicates that the linear system is stable. This is also supported by the pole-zero map where all the poles are located at the left hand side of $y$-axis. In particular, the obtained values of poles are $-36.6623,-0.2344 \pm j 14.5053$ and -1.4828 , while the obtained values of zeros are $4.7468,0.0274 \pm j 0.0661$. On the other hand, we also can observe the feature of nonlinear function by plotting the obtained piece-wise function as depicted in Figure 10. Note that our nonlinear function is not restricted to any form of nonlinear function (i.e., quadratic), which is more generalized and provide more flexibility of searching a justifiable function.

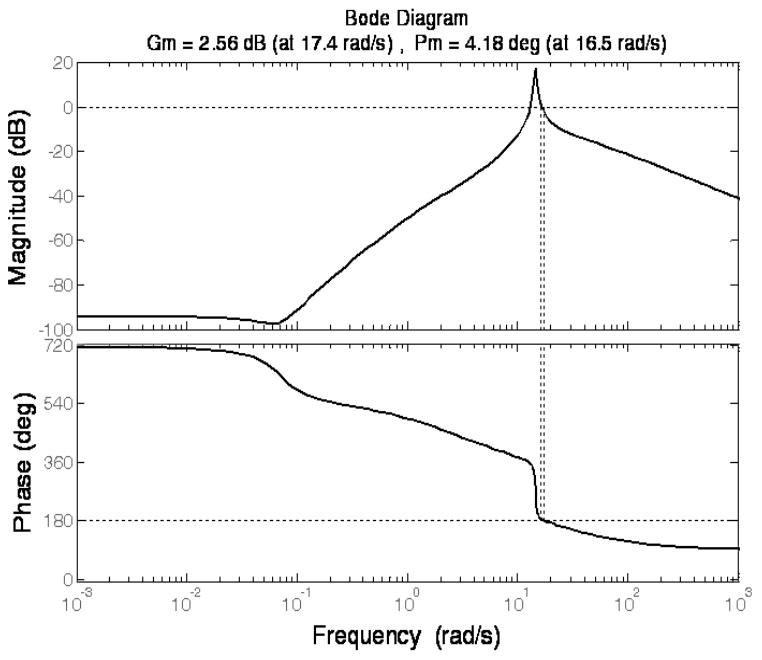

Figure 8. Bode plot of transfer function $G(s)$



Figure 9. Pole-zero map of transfer function $G(s)$ 


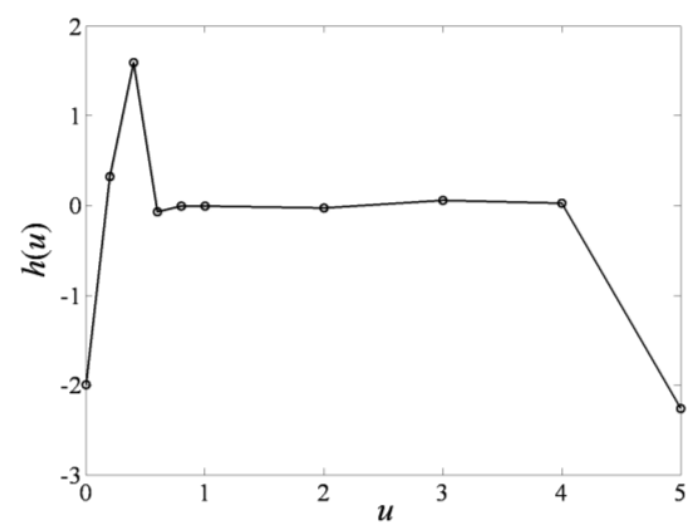

Figure 10. Resultant of piece-wise affine function $h(u)$

\section{CONCLUSION}

In this paper, an identification of liquid slosh plant using continuous-time Hammerstein model based on Grey Wolf Optimizer (GWO) has been presented. The results demonstrated that the proposed generic Hammerstein model based on GWO has a good potential in identifying the real liquid slosh behavior. In particular, it is shown that the proposed method is able to produce a quite close identified output with real liquid slosh output. Moreover, the resultant linear model has been proved to be stable based on the bode plot and pole-zero map. It is also shown that the used of piecewise-affine function gives more flexibility for the GWO to search more generic nonlinear function. In the future, our work can be extended to various types of nonlinear function such as continuous-time Wiener and Hammerstein-Wiener.

\section{ACKNOWLEDGEMENTS}

The authors gratefully acknowledged Research and Innovation Department of Universiti Malaysia Pahang under grant RDU1703153 for the financial support.

\section{REFERENCES}

[1] E. Rizzuto and R. Tedeschi, "Surveys of actual sloshing loads on board of ships at sea," in Proceedings of International Conference on Ship and Marine Research, 1997, pp. 7.29-7.37.

[2] K. Terashima and G. Schmidt, "Sloshing analysis and suppression control of tilting-type automatic pouring machine," in Proceedings of IEEE International Symposium on Industrial Electronics, 1994, pp. 275-280.

[3] T. Acarman and U. Ozguner, "Rollover prevention for heavy trucks using frequency shaped sliding mode control," Vehicle System Dynamics, vol. 44, no. 10, pp. 737-762, 2006.

[4] C. Li, et al., "Identification of the Hammerstein model of a PEMFC stack based on least squares support vector machines,"Journal of Power Sources, vol. 175, pp. 303-316, 2008.

[5] T. Kara and I. Eker, "Nonlinear modeling and identification of a DC motor for bidirectional operation with real time experiments," Energy Conversion and Management, vol. 45, no. 7-8, pp. 1087-1106, 2004.

[6] S. W. Su, et al., "Oxygen uptake estimation in humans during exercise using a Hammerstein model," Annals of biomedical engineering, vol. 35, no. 11, pp. 1898-1906, 2007.

[7] D. T. Westwick and R.E. Kearney, "Separable least squares identification of nonlinear Hammerstein models: Application to stretch reflex dynamics," Annals of Biomedical Engineering, vol. 29, no. 8, pp. 707-718, 2001.

[8] Q. Zhang, et al., "Nonlinear modeling and predictive functional control of Hammerstein system with application to the turntable servo system," Mechanical Systems and Signal Processing, vol. 72, pp. 383-394, 2016.

[9] Q. Ai, et al., "Hammerstein model for hysteresis characteristics of pneumatic muscle actuators," International Journal of Intelligent Robotics and Applications, vol. 3, no. 1, pp. 33-44, 2019.

[10] A. Saleem, et al., "Nonlinear Hammerstein model identification of amplified piezoelectric actuators (APAs): Experimental considerations,"in 4th International Conference on Control, Decision and Information Technologies (CoDIT), pp. 0633-0638, 2017.

[11] H. T. Zhang, et al., "Distributed Hammerstein Modeling for Cross-Coupling Effect of Multiaxis Piezoelectric Micropositioning Stages," IEEE/ASME Transactions on Mechatronics, vol. 23, no. 6, pp. 2794-2804, 2018.

[12] E. W. Bai and D. Li, "Convergence of the iterative Hammerstein system identification algorithm," IEEE Transactions on automatic control, vol. 49, no. 11, pp. 1929-1940, 2004.

[13] J. Hou, et al., "Fixed point iteration-based subspace identification of Hammerstein state-space models," IET Control Theory \& Applications, vol. 13, no. 8, pp. 1173-1181, 2019. 
[14] Z. Ge, et al., "Gradient-based iterative identification method for multivariate equation-error autoregressive moving average systems using the decomposition technique," Journal of the Franklin Institute, vol. 356, no. 3, pp. 1658-1676, 2019.

[15] J. Hou, et al., "Subspace Hammerstein model identification under periodic disturbance," IFAC-PapersOnLine, vol. 51, no. 15, pp. 335-340, 2018.

[16] J. Hou, et al., "Subspace identification of Hammerstein-type nonlinear systems subject to unknown periodic disturbance," International Journal of Control, pp. 1-29, 2019.

[17] I.W. Jamaludin and N.A. Wahab, "Recursive Subspace Identification Algorithm using the Propagator Based Method,"Indonesian Journal of Electrical Engineering and Computer Science, vol. 6, no. 1, pp. 172-179, 2017.

[18] D. Wang and W. Zhang, "Improved least squares identification algorithm for multivariable Hammerstein systems,"Journal of the Franklin Institute, vol. 352, no. 11, pp. 5292-5307, 2015.

[19] E. W. Bai, "A blind approach to the Hammerstein-Wiener model identification," Automatica, vol. 38, no. 6, pp. 967-979, 2002.

[20] L. Ma and X. Liu, "A nonlinear recursive instrumental variables identification method of Hammerstein ARMAX system," Nonlinear Dynamics, vol. 79, no. 2, pp. 1601-1613, 2015.

[21] W. Lin and P.X. Liu, "Hammerstein model identification based on bacterial foraging," Electronics Letters, vol. 42, no. 23, pp. 1332-1333, 2006.

[22] A. Gotmare, et al., "Nonlinear system identification using a cuckoo search optimized adaptive Hammerstein model," Expert systems with applications, vol. 42, no. 5, pp. 2538-2546, 2015.

[23] H. N. Al-Duwaish, "Identification of Hammerstein models with known nonlinearity structure using particle swarm optimization," Arabian Journal for Science and Engineering, vol. 36, no. 7, pp. 1269-1276, 2011.

[24] H. Zhang and H. Zhang, "Identification of Hammerstein Model Based on Quantum Genetic Algorithm,"Telecommunication Computing Electronics and Control, vol. 11, no. 12, pp. 7206-7212, 2013.

[25] S. Mirjalili, et al., "Grey wolf optimizer," Advances in engineering software, vol. 69, pp. 46-61, 2014.

[26] Y. L. Karnavas, et al., "Permanent magnet synchronous motor design using grey wolf optimizer algorithm," International Journal of Electrical and Computer Engineering, vol. 6, no. 3, pp. 1353-1362, 2016.

[27] M. Z. Mohd Tumari, M. H. Suid, and M. A. Ahmad, "A modified Grey Wolf Optimizer for improving wind plant energy production,"Indonesian Journal of Electrical Engineering and Computer Science, vol. 18, no. 3, pp. 1123-1129, June 2020. 